\title{
Cardiac power output accurately reflects external cardiac work over a wide range of inotropic states in pigs
}

Dawud Abawi ${ }^{1}$, Alessandro Faragli ${ }^{1,2,3}$, Michael Schwarz ${ }^{4,5}$, Martin Manninger ${ }^{6}$, David Zweiker ${ }^{6}$, Karl-Patrik Kresoja ${ }^{7,8}$, Jochen Verderber ${ }^{6}$, Birgit Zirngast ${ }^{9}$, Heinrich Maechler ${ }^{9}$, Paul Steendijk ${ }^{10}$, Burkert Pieske ${ }^{1,2,3,11}$, Heiner Post ${ }^{1,2,3,12}$ and Alessio Alogna $a^{1,2,3^{*}}$ (D)

\begin{abstract}
Background: Cardiac power output (CPO), derived from the product of cardiac output and mean aortic pressure, is an important yet underexploited parameter for hemodynamic monitoring of critically ill patients in the intensivecare unit (ICU). The conductance catheter-derived pressure-volume loop area reflects left ventricular stroke work (LV SW). Dividing LV SW by time, a measure of LV SW $\mathrm{min}^{-1}$ is obtained sharing the same unit as CPO (W). We aimed to validate CPO as a marker of LV SW min $^{-1}$ under various inotropic states.

Methods: We retrospectively analysed data obtained from experimental studies of the hemodynamic impact of mild hypothermia and hyperthermia on acute heart failure. Fifty-nine anaesthetized and mechanically ventilated closed-chest Landrace pigs $(68 \pm 1 \mathrm{~kg})$ were instrumented with Swan-Ganz and LV pressure-volume catheters. Data were obtained at body temperatures of $33.0^{\circ} \mathrm{C}, 38.0^{\circ} \mathrm{C}$ and $40.5^{\circ} \mathrm{C}$; before and after: resuscitation, myocardial infarction, endotoxemia, sevoflurane-induced myocardial depression and beta-adrenergic stimulation. We plotted LVSW min $^{-1}$ against CPO by linear regression analysis, as well as against the following classical indices of LV function and work: LV ejection fraction (LV EF), rate-pressure product (RPP), triple product (TP), LV maximum pressure $\left(L V P_{\text {max }}\right)$ and maximal rate of rise of $L V P\left(L V d P / d t_{\text {max }}\right)$.
\end{abstract}

Results: CPO showed the best correlation with LV SW $\min ^{-1}\left(r^{2}=0.89 ; p<0.05\right)$ while LV EF did not correlate at all $\left(r^{2}=0.01 ; p=0.259\right)$. Further parameters correlated moderately with LV SW $\min ^{-1}\left(\operatorname{LVP} \max r^{2}=0.47, \operatorname{RPP} r^{2}=0.67\right.$; and $\left.\mathrm{TP} r^{2}=0.54\right)$. LV dP/dt $t_{\max }$ correlated worst with LV SW $\min ^{-1}\left(r^{2}=0.28\right)$.

Conclusion: CPO reflects external cardiac work over a wide range of inotropic states. These data further support the use of CPO to monitor inotropic interventions in the ICU.

Keywords: Acute heart failure, Cardiac power output, Cardiogenic shock, Ejection fraction, Stroke work

\section{Background}

Benefits of acquiring hemodynamic information by pulmonary artery catheterization (PAC) in conditions of critical illness are controversial. Following several neutral studies arguing against the survival benefit of hemodynamic monitoring by PAC in different populations of critically ill patients [1-9], the use of PAC is not recommended as a

\footnotetext{
* Correspondence: alessio.alogna@charite.de

'Department of Internal Medicine and Cardiology, Charité -

Universitätsmedizin Berlin, Campus Virchow-Klinikum, Augustenburgerplatz 1, 13353 Berlin, Germany

${ }^{2}$ Berlin Institute of Health (BIH), Berlin, Germany

Full list of author information is available at the end of the article
}

diagnostic routine anymore $[10,11]$. One possible explanation for the neutral effects of PAC is the lack of clear hemodynamic goals to guide evidence-based therapies [4]. A promising parameter for hemodynamic monitoring of critically ill patients is cardiac power output (CPO), which is assessed as the product of cardiac output (CO) and mean aortic pressure (MAP), divided by a constant of 451 [12]. Being the product of flow and pressure, $\mathrm{CPO}$ describes the function of the heart as a mechanical pump [13], representing the rate of external work done by the left ventricle. In addition, CPO has been shown to be the strongest independent predictor of intrahospital mortality in patients with

(c) The Author(s). 2019 Open Access This article is distributed under the terms of the Creative Commons Attribution 4.0 International License (http://creativecommons.org/licenses/by/4.0/), which permits unrestricted use, distribution, and reproduction in any medium, provided you give appropriate credit to the original author(s) and the source, provide a link to the Creative Commons license, and indicate if changes were made. The Creative Commons Public Domain Dedication waiver (http://creativecommons.org/publicdomain/zero/1.0/) applies to the data made available in this article, unless otherwise stated. 
cardiogenic shock [12] and to strongly correlate with outcome in chronic heart failure patients [14]. CPO might therefore be a relevant yet underexplored way to describe a patient's hemodynamic state in the intensive care unit (ICU) setting [12].

The most comprehensive way to describe ventricular performance is pressure-volume analysis. Over the last decades, it became the gold standard to quantify cardiac function in vivo by providing measures that are reasonably loadindependent, such as the end-systolic and end-diastolic pressure-volume relationships [15-17]. Pressure-volume analysis not only offers load-independent information, but it allows to quantify ventricular energetics and describes the interaction between heart and vasculature [18-20]. The area of the pressure-volume loop reflects the external work of the heart, also called left ventricular stroke work (LV SW). When LV SW is divided by time, a measure of LV SW per minute is obtained that shares the same unit as $\mathrm{CPO}$ (Watts). The correlation between $\mathrm{CPO}$, as measured by PAC and invasive arterial pressure, and LV SW $\mathrm{min}^{-1}$ measured via the invasive gold-standard (conductance method) has never been tested so far. The aim of the study was therefore to validate $\mathrm{CPO}$ as a marker of the actual LV SW $\mathrm{min}^{-}$ ${ }^{1}$ over a wide range of inotropic states. We therefore conducted a retrospective data analysis of previous animal studies from our group, in which we investigated the hemodynamic impact of mild hypothermia $\left(\mathrm{MH}, 33.0^{\circ} \mathrm{C}\right)$ and hyperthermia $\left(\mathrm{HT}, 40.5^{\circ} \mathrm{C}\right.$ ) by pressure-volume analysis in several porcine models of acute heart failure representative for patients in the ICU [21-24]. LV SW $\mathrm{min}^{-1}$ was further compared with clinically established indices to describe LV function and work, such as left ventricular ejection fraction (LV EF), left ventricular maximum pressure $\left(\mathrm{LVP}_{\max }\right)$, maximum rate of rise of left ventricular pressure (LV dP/ $\left.\mathrm{dt}_{\max }\right)$, rate-pressure product $[\mathrm{RPP}=$ heart rate $(\mathrm{HR}) \mathrm{x}$ $\left.\mathrm{LVP}_{\max }\right]$ and triple product $\left(\mathrm{TP}=\mathrm{RPP} \times \mathrm{LV} \mathrm{dP} / \mathrm{dt}_{\max }\right)$.

\section{Methods}

The experimental protocols were approved by the local bioethics committee of Vienna, Austria (Austrian Committee for Animal Trials, in German "Tierversuchskommission", BMWF-66.010/0091-II/3b/2013, BMWF66.010/0033-II/10b/2008, BMWF-66.010/0103-II/10b/ 2009, BMWF-66.010/0108-II/3b/2010), and conform to the "European Convention for the Protection of Vertebrate Animals used for Experimental and other Scientific Purposes" (Council of Europe No 123, Strasbourg 1985). Landrace pigs were ordered from Heinz Stelzl, Großklein, Austria, and were delivered to the Institute for Biomedical Research of the Medical University of Graz (Roseggerweg 48, 8036 Graz, Austria). The experiments were performed either at the Section for Surgical Research, Department of Experimental Surgery (Auenbruggerplatz 15, 8036 Graz, Austria) or at the abovementioned Institute for Biomedical Research of the Medical University of Graz.

\section{Experimental setup}

The experimental setup has been described before [25]. Briefly, Landrace pigs $(n=59,68 \pm 1 \mathrm{~kg})$ were fasted overnight with free access to water and sedated with $0.25-0.5$ $\mathrm{mg} \mathrm{kg}^{-1}$ midazolam (Midazolam „ERWO “ $5 \mathrm{mg} / \mathrm{ml}$ - ampoules, ERWO Pharma GmbH, Brunn am Gebirge, Austria) and 10-20 $\mathrm{mg} \mathrm{kg}^{-1}$ ketamine (Ketasol $100 \mathrm{mg} / \mathrm{ml}$, aniMedica $\mathrm{GmbH}$, Senden-Bösensell, Germany). The animals were intubated and anaesthesia was continued with 1.0-2.0 Vol\% sevoflurane (Sevorane ${ }^{\circ}$, Abbott GmbH, Vienna, Austria), 30 $35 \mathrm{\mu g} \mathrm{kg}^{-1} \mathrm{~h}^{-1}$ fentanyl (Fentanyl-Janssen ${ }^{\mathrm{m}}$, $0.1 \mathrm{mg}$ ampoules, Janssen-Cilag Pharma, Vienna, Austria), $1-1.25 \mathrm{mg} \mathrm{kg}^{-1} \mathrm{~h}^{-1}$ midazolam, $3 \mathrm{mg} \mathrm{kg}^{-1} \mathrm{~h}^{-1}$ ketamine and $0.2 \mathrm{mg} \mathrm{kg}^{-1} \mathrm{~h}^{-1}$ pancuronium (Pancouronium bromide $2 \mathrm{mg} / \mathrm{ml}$ ampoules, Ratiopharm GmbH, Ulm, Germany). Pigs were ventilated (Julian, Draeger, Vienna, Austria) with an $\mathrm{FiO}_{2}$ (Fraction of inspired oxygen) of 0.5 , an I: E-ratio of 1:1.5, the positive end-expiratory pressure was set at $5 \mathrm{mmHg}$ and a tidal volume (VT) of $10 \mathrm{ml} \mathrm{kg}^{-1}$. The respiratory rate was adjusted constantly to maintain an end-expiratory carbon dioxide partial pressure between 35 and $45 \mathrm{mmHg}$. Under fluoroscopic guidance, all animals were instrumented with a Swan-Ganz catheter (Edwards Lifesciences CCO connected to Vigilance I, Edwards Lifesciences, Irvine, CA, USA), and an LV conductance catheter (5F, 12 electrodes, $7 \mathrm{~mm}$ spacing, MPVS Ultra, Millar Instruments, Houston, Texas, USA). A 14-F sheath was introduced into the left femoral vein, and an intravascular cooling catheter connected to a cooling unit (Accutrol $^{\text {Tm }}$ Catheter 14F and InnerCool RTx Endovascular System, Philips Healthcare, Vienna, Austria) was positioned with the tip at the level of the diaphragm in the inferior caval vein. The body core temperature was measured at the tip of the Swan-Ganz-catheter. After instrumentation, the animals were allowed to stabilize for $30-60 \mathrm{~min}$.

\section{Experimental protocols}

The experimental protocols are summarized in Fig. 1 and have been described in detail before [21-24].

Steady-state hemodynamics were obtained and averaged over three respiratory cycles. At each time point, volumetric conductance data were calibrated by measuring cardiac output (slope factor a) and hypertonic (10\%) saline infusion (parallel volume) as described earlier [26, 27]. At the end of each experimental protocol anaesthetised and unconscious animals were euthanized by an injection of an $80 \mathrm{mmol}$ potassium chloride bolus.

\section{Group 1 - Resuscitation after ventricular fibrillation (RES)}

Via a pacemaker lead, ventricular fibrillation (VF) was induced by applying an alternating current $(50 \mathrm{~Hz})$. After VF sustained for $5 \mathrm{~min}$, resuscitation was begun by 
a)

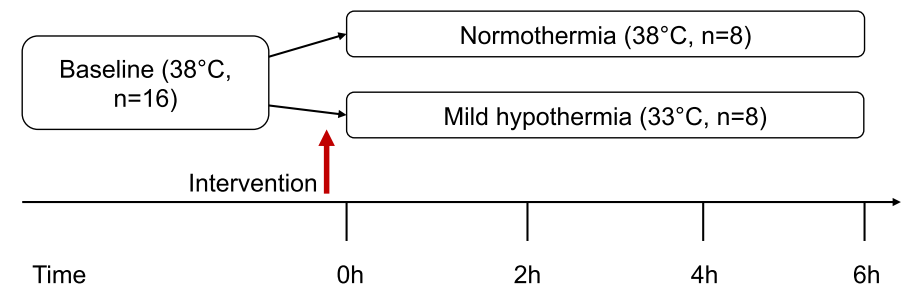

b)

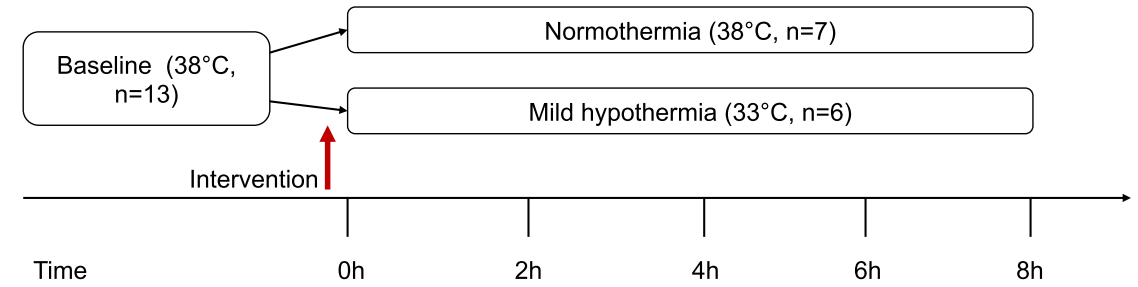

c)

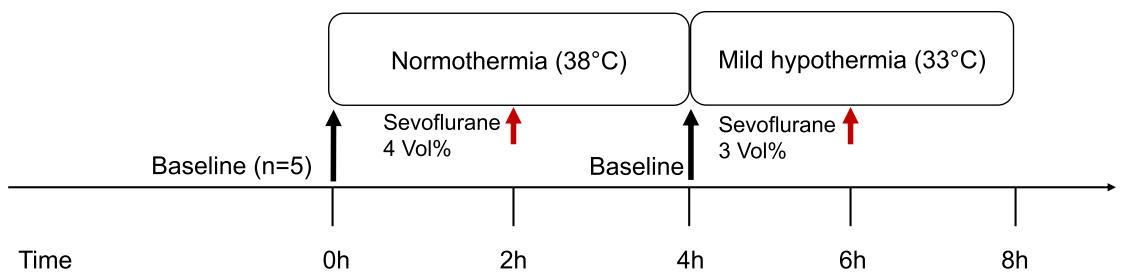

d)

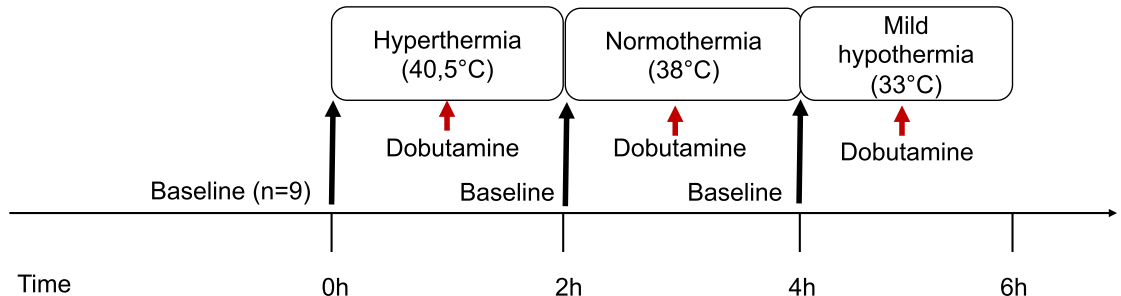

Fig. 1 Experimental protocols: a Includes group 1-2 which are described as follows: group 1: resuscitation after ventricular fibrillation (RES, total $n=16$ ), group 2: myocardial infarction by coronary microembolisation (CME, total $n=16$ ); $\mathbf{b}$ group 3: endotoxemia by LPS-infusion (LPS, total $n=$ 13); c group 4: sevoflurane-induced myocardial depression (SEVO, total $n=5)$; $\mathbf{d}$ group 5: Temperature modulation from hyperthermia to mild hypothermia vs dobutamine (DOB, total $n=9)$

manual external chest compression at $80 \mathrm{~min}^{-1}$, a single bolus of $15 \mathrm{mg} \mathrm{kg}^{-1}$ adrenaline and biphasic defibrillation (200 J, Responder 2000; General Electric, Fairfield, CT, USA). Pigs (total $n=16$ ) were assigned in a sequential $1: 1$ fashion to either normothermia (NT, $\left.n=8,38.0^{\circ} \mathrm{C}\right)$ or $\mathrm{MH}\left(n=8,33.0^{\circ} \mathrm{C}\right)$ as shown in Fig. 1 a. After $10 \mathrm{~min}$ past ROSC (return of spontaneous circulation), additional volume was given at either room temperature to normothermic animals or pre-cooled at $4{ }^{\circ} \mathrm{C}$ to cooled animals, consisting of $16 \mathrm{ml} \mathrm{kg}^{-1}$ crystalloid solution and $8 \mathrm{ml} \mathrm{kg}^{-1}$ hydroxyethyl starch (Voluven 6\% 130/0.4; Fresenius, Austria) due to a substantial intravasal fluid loss. No further inotropes were given after ROSC [21]. For the following data analysis, the timepoints baseline and $6 \mathrm{~h}$ after return of spontaneous circulation were used.

\section{Group 2 - Myocardial infarction after coronary microembolisation (CME)}

Through repetitive slow (1 min) injections of 500,000 polystyrene microspheres $(45 \mu \mathrm{m})$ into the proximal left circumflex coronary artery, myocardial infarction was induced. The injections were continued until cardiac power output was reduced by more than $40 \%$. Three pigs developed sustained ventricular fibrillation during the injections and were excluded from the following analysis. The remaining animals (total $n=16$ ) were assigned in a sequential 1:1 fashion to either NT $\left(n=8,38.0^{\circ} \mathrm{C}\right)$ or $\mathrm{MH}$ $\left(n=8,33.0^{\circ} \mathrm{C}\right)$ as shown in Fig. 1a [22]. We used baseline and $6 \mathrm{~h}$ after $\mathrm{CME}$ as timepoints for the data analysis.

\section{Group 3 - Endotoxemia after LPS-infusion (LPS)}

Following a Lipopolysaccharide (LPS)-infusion of $4 \mathrm{~h}$, pigs (total $n=13$ ) were monitored for another $4 \mathrm{~h}$, 
resulting in a total of $8 \mathrm{~h}$ [23]. Assignment to either NT $\left(n=7,38.0^{\circ} \mathrm{C}\right)$ or $\mathrm{MH}\left(n=6,33.0^{\circ} \mathrm{C}\right)$ in a sequential $1: 1$ fashion started with the beginning of LPS-infusion (Fig. $1 \mathrm{~b})$. With the onset of LPS-infusion cooling was started simultaneously in $\mathrm{MH}$ by the intravascular device and infusion of pre-cooled $\left(4^{\circ} \mathrm{C}\right)$ instead of a crystalloid solution at room temperature $\left(10 \mathrm{ml} \mathrm{kg}^{-1} \mathrm{~h}^{-1}\right.$ in both groups). When MAP fell below $55 \mathrm{mmHg}$ during LPSinfusion, animals received up to four additional boluses of $500 \mathrm{ml}$ crystalloid infusion, of which the last two were enriched with $1 \mu \mathrm{g} \mathrm{kg}^{-1}$ epinephrine. When $\mathrm{S}_{\mathrm{a}} \mathrm{O}_{2}$ (arterial oxygen saturation) fell below $90 \%, \mathrm{FiO}_{2}$ was increased to 1.0 and the I: $\mathrm{E}$ ratio was changed to $1: 1$. When peak respiratory pressure reached $35 \mathrm{mmHg}$, endotracheal tube suction was performed, plus the tidal volume was reduced to $8 \mathrm{ml} \mathrm{kg}^{-1}$ with a compensatory increase in respiration rate. For the following data analysis timepoints baseline and $8 \mathrm{~h}$ after onset of LPS infusion were used.

\section{Group 4 - Sevoflurane-induced myocardial depression (SEVO)}

After baseline measurements at NT $\left(n=5,38.0^{\circ} \mathrm{C}\right)$, the continuous administration of sevoflurane was elevated from a baseline of 1.0-2.0 to 4 Vol\%. Subsequently, the continuous administration of sevoflurane was reduced to baseline values, and animals were cooled to $\mathrm{MH}\left(33.0^{\circ} \mathrm{C}\right)$. After new baseline measurements in the same animals at $\mathrm{MH}$, the continuous administration of sevoflurane was elevated from $1.0-2.0$ to $3 \mathrm{Vol} \%$. The Vol\% of sevoflurane to induce cardiac depression during NT and $\mathrm{MH}$ was established in a series of pilot experiments. The sevoflurane dose was titrated aiming at an approximate $50 \%$ reduction of $\mathrm{LV} \mathrm{dP/}$ $\mathrm{dt}_{\max }$. A lower Vol\% of sevoflurane was needed to induce the same magnitude of depression of contractile function during $\mathrm{MH}$, probably due to different pharmacokinetics of the anesthetics at lower body temperatures, as previously reported [28-32]. The abovementioned timepoints (baseline at NT, 4 Vol\% sevoflurane, baseline at $\mathrm{MH}$ and $3 \mathrm{Vol} \%$ sevoflurane) were considered for data analysis (Fig. 1c).

\section{Group 5 - Temperature modulation from hyperthermia to mild hypothermia vs dobutamine (DOB)}

A patient warming system (Bair Hugger, Warmtouch Series 500/OR, 3 M, Germany) with warming cover was used to increase and maintain the animals (total $n=9$ ) body temperature at $40.5^{\circ} \mathrm{C}$ (hyperthermia, HT). Only animals in this group were subsequently cooled from HT to NT and MH; at each temperature step baseline measurements were followed by a dobutamine-stress protocol until the steady state $\mathrm{LV} \mathrm{dP} / \mathrm{dt}_{\max }$ was doubled (Fig. 1d) [24]. The dose of dobutamine necessary to double $\mathrm{LV} \mathrm{dP} / \mathrm{dt}_{\max }$ decreased along with temperature from $2.1 \pm 0.1 \mu \mathrm{g} \mathrm{kg}^{-1} \mathrm{~min}^{-1}$ (hyperthermia) to $1.8 \pm$
$0.1 \mu \mathrm{g} \mathrm{kg}^{-1} \mathrm{~min}^{-1}$ (normothermia) and further to $1.5 \pm$ $0.1 \mu \mathrm{g} \mathrm{kg}^{-1} \mathrm{~min}^{-1}$ (mild hypothermia). Baseline measurements at each temperature step and the corresponding measurements during dobutamine infusion were considered for data analysis.

\section{Data processing and statistical analyses}

Details on data analysis have been described before [25]. Pressure-volume data and time intervals were analysed offline by CircLab Software (custom made by P. Steendijk). End-diastole was defined as the time-point of zero crossing of $\mathrm{LV} \mathrm{dP} / \mathrm{dt}$ before its rapid upstroke. End-systole was defined as the time point of maximum pressure/volume ratio. CPO (W), LV SW $\min ^{-1}(\mathrm{~W}), \mathrm{LV}$ EF, RPP and TP were calculated by using the following equations:

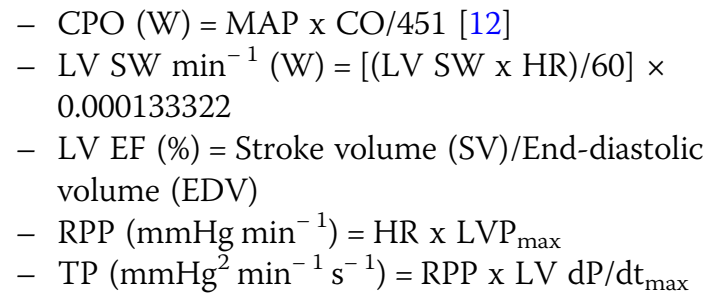

All data are presented as mean \pm standard deviation (SD). The correlations between LV SW and LV SW $\mathrm{min}^{-1}$ with $\mathrm{CPO}, \mathrm{LV} \mathrm{EF}, \mathrm{RPP}, \mathrm{TP}, \mathrm{LVP}_{\max }$ and $\mathrm{LV} \mathrm{dP} / \mathrm{dt}_{\max }$ were assessed by linear regression analysis. The assumption of normality of residuals was checked by the KolmogorovSmirnov test as well as graphically by a probability plot. Data between groups before and after cardiac insult at different temperatures were analysed by two-way ANOVA (groups 1-3). A one-way ANOVA for repeated measurements was used to compare data within one group (group 4). Steady state data at different temperatures, before and during dobutamine infusion, were compared by two-way ANOVA for repeated measurements (group 5). Post-hoc testing was performed by Tukey's test. Normality was demonstrated by the Shapiro-Wilks test or by visual inspection of normal probability plots. Nonnormally distributed variables were examined either by Kruskal-Wallis- or Friedman-Test. A $p$-value $<0.05$ was considered significant. For statistical calculations, we used the software Sigmastat (Version 4.0, Systat Software, Inc) and SPSS (Version 23.0, IBM, Armonk, NY).

\section{Results}

\section{Systemic hemodynamics}

Systemic hemodynamics from the experimental protocols are summarized in Table 1. CO, LV SW $\mathrm{min}^{-1}$ and CPO spanned over a wide range of values, with the following means (ranges): CO $5.7 \mathrm{~L} \mathrm{~min}^{-1}\left(8.2 \mathrm{~L} \mathrm{~min}^{-1}\right)$, CPO 0.94 $\mathrm{W}(1.75 \mathrm{~W})$ and LV SW $\mathrm{min}^{-1} 0.99 \mathrm{~W}(1.88 \mathrm{~W})$.

During mild hypothermia, heart rate decreased $(p<0.05)$ in all animals and was significantly lower at $\mathrm{MH}$ than 
Table 1 Systemic hemodynamics

\begin{tabular}{|c|c|c|c|c|c|c|c|}
\hline Hemodynamic Parameter & Temp. & Timepoint & RES & CME & LPS & SEVO & Dob \\
\hline $\mathrm{HR}$ & HT & Baseline & & & & & $98 \pm 12.8$ \\
\hline \multirow[t]{5}{*}{$\left(1 \min ^{-1}\right)$} & & After Intervention & & & & & $122 \pm 10.1^{c}$ \\
\hline & NT & Baseline & $89 \pm 10.6$ & $89 \pm 11.8$ & $97 \pm 10$ & $90 \pm 14.7$ & $89 \pm 13.2$ \\
\hline & & After Intervention & $84 \pm 13.2$ & $101 \pm 18.5^{*}$ & $128 \pm 17.4^{*}$ & $79 \pm 9.6^{*}$ & $114 \pm 10.2^{a, c}$ \\
\hline & $\mathrm{MH}$ & Baseline & $86 \pm 16.7$ & $88 \pm 10.8$ & $98 \pm 11.3$ & $70 \pm 10.1$ & $65 \pm 8^{a, b}$ \\
\hline & & After Intervention & $59 \pm 10.7^{* t}$ & $67 \pm 11.4^{* t}$ & $79 \pm 11.6^{* t}$ & $60 \pm 5.9^{*}$ & $83 \pm 11.2^{a, b, c}$ \\
\hline MAP & $\mathrm{HT}$ & Baseline & & & & & $74 \pm 8.4$ \\
\hline \multirow[t]{5}{*}{$(\mathrm{mmHg})$} & & After Intervention & & & & & $77 \pm 12.3$ \\
\hline & NT & Baseline & $92 \pm 11.7$ & $104 \pm 17.3$ & $86 \pm 4.6$ & $75 \pm 7.1$ & $68 \pm 8$ \\
\hline & & After Intervention & $64 \pm 8.2^{*}$ & $54 \pm 13^{*}$ & $53 \pm 10.6^{*}$ & $44 \pm 4.9^{*}$ & $71 \pm 11.8$ \\
\hline & $\mathrm{MH}$ & Baseline & $90 \pm 8.7$ & $107 \pm 10.3$ & $85 \pm 5.9$ & $67 \pm 7.7$ & $65 \pm 7.7^{\mathrm{a}}$ \\
\hline & & After Intervention & $73 \pm 15.4^{*}$ & $69 \pm 6.7^{* t}$ & $58 \pm 2.9^{*}$ & $42 \pm 3.3^{*}$ & $69 \pm 6.5^{\mathrm{a}}$ \\
\hline $\mathrm{CO}$ & $\mathrm{HT}$ & Baseline & & & & & $6.7 \pm 0.9$ \\
\hline \multirow[t]{5}{*}{$\left(L \min ^{-1}\right)$} & & After Intervention & & & & & $8.4 \pm 1^{c}$ \\
\hline & NT & Baseline & $5.7 \pm 1.2$ & $6.2 \pm 0.9$ & $6.8 \pm 0.5$ & $6.7 \pm 0.6$ & $6.1 \pm 1$ \\
\hline & & After Intervention & $4.8 \pm 1.1$ & $3.5 \pm 0.7^{*}$ & $6.6 \pm 1.3$ & $5.0 \pm 0,8^{*}$ & $7.8 \pm 1.1^{c}$ \\
\hline & $\mathrm{MH}$ & Baseline & $5.4 \pm 1.3$ & $6.5 \pm 0.9$ & $6.2 \pm 0.8$ & $4.9 \pm 0.6$ & $4.4 \pm 0.6^{a, b}$ \\
\hline & & After Intervention & $3.5 \pm 0.7^{* t}$ & $3.2 \pm 0.5^{*}$ & $4.5 \pm 1.22^{* t}$ & $4.4 \pm 0.6$ & $6.0 \pm 0.85^{a, b, c}$ \\
\hline SVR & $\mathrm{HT}$ & Baseline & & & & & $842 \pm 152$ \\
\hline \multirow[t]{5}{*}{ (dyne.sec $\mathrm{cm}^{-5}$ ) } & & After Intervention & & & & & $717 \pm 170^{c}$ \\
\hline & NT & Baseline & $1238 \pm 401$ & $1292 \pm 345$ & $931 \pm 76$ & $848 \pm 97$ & $856 \pm 171$ \\
\hline & & After Intervention & $915 \pm 219$ & $1089 \pm 256$ & $531 \pm 83^{*}$ & $620 \pm 117^{*}$ & $703 \pm 128^{c}$ \\
\hline & $\mathrm{MH}$ & Baseline & $1252 \pm 310$ & $1260 \pm 153$ & $1018 \pm 139$ & $1030 \pm 137$ & $1154 \pm 202^{a, b}$ \\
\hline & & After Intervention & $1470 \pm 384^{+}$ & $1618 \pm 252^{* t}$ & $884 \pm 206^{t}$ & $675 \pm 183^{*}$ & $880 \pm 88^{a, b, c}$ \\
\hline CPO & $\mathrm{HT}$ & Baseline & & & & & $1.10 \pm 0.2$ \\
\hline \multirow[t]{5}{*}{$(W)$} & & After Intervention & & & & & $1.40 \pm 0.3^{c}$ \\
\hline & NT & Baseline & $1.14 \pm 0.2$ & $1.43 \pm 0.3$ & $1.29 \pm 0.1$ & $1.12 \pm 0.2$ & $0.90 \pm 0.2$ \\
\hline & & After Intervention & $0.69 \pm 0.24^{*}$ & $0.42 \pm 0.2^{*}$ & $0.80 \pm 0.3^{*}$ & $0.48 \pm 0.1^{*}$ & $1.20 \pm 0.3^{a, c}$ \\
\hline & $\mathrm{MH}$ & Baseline & $1.09 \pm 0.3$ & $1.55 \pm 0.3$ & $1.17 \pm 0.2$ & $0.72 \pm 0.1$ & $0.60 \pm 0.1^{a, b}$ \\
\hline & & After Intervention & $0.58 \pm 0.2^{*}$ & $0.49 \pm 0.1^{*}$ & $0.58 \pm 0.2^{*}$ & $0.40 \pm 0.0^{*}$ & $0.90 \pm 0.2^{a, b, c}$ \\
\hline
\end{tabular}

All values are presented as mean \pm standard deviation (SD). Temp. Temperature, HT Hyperthermia, NT Normothermia, MH Mild hypothermia, RES Resuscitation after ventricular fibrillation, CME Coronary microembolisation, LPS Endotoxemia by Lipopolysaccharide (LPS)-infusion, SEVO Sevoflurane-induced myocardial depression, Dob Temperature modulation from hyperthermia to mild hypothermia vs. dobutamine (intervention) at each temperature step, HR Heart rate, MAP Mean aortic pressure, CO Cardiac Output, SVR Systemic vascular resistance, CPO Cardiac Power Output

${ }^{*} p<0.05$ vs baseline; ${ }^{\dagger} p<0.05$ vs NT; ${ }^{a} p<0.05$ vs hyperthermia; ${ }^{b} p<0.05$ vs normothermia; ${ }^{c} p<0.05$ vs baseline at each temperature step

during normothermia or hyperthermia after each intervention. Dobutamine infusion increased heart rate at each temperature step.

Mean aortic pressure decreased with all interventions and was more preserved during $\mathrm{MH}$ after myocardial infarction $(p<0.05)$.

In group 5 (DOB), $\mathrm{CO}$ and $\mathrm{CPO}$ decreased with cooling from hyperthermia to normothermia to $\mathrm{MH}$ and were increased by dobutamine at each temperature step (Table 1). In groups $1-4, \mathrm{CO}$ was per se significantly lower during $\mathrm{MH}$ than during NT. $\mathrm{CPO}$ decreased significantly with the interventions in groups 1-4, but there was no significant difference between NT and MH.

Systemic vascular resistance increased with cooling from hyperthermia to $\mathrm{MH}$ and was decreased by dobutamine at each temperature step. Except for sevoflurane-induced myocardial depression, SVR was significantly higher during $\mathrm{MH}$ after each intervention.

Cardiac power output and other parameters of cardiac function as indices of left ventricular stroke work CPO showed the best correlation with LV SW $\min ^{-1}$ (Fig. 2; $\left.r^{2}=0.89 ; p<0.001\right)$. LV EF did not correlate at all, neither 


\section{CPO (W) vs. LV SW $\min ^{-1}$ (W)}

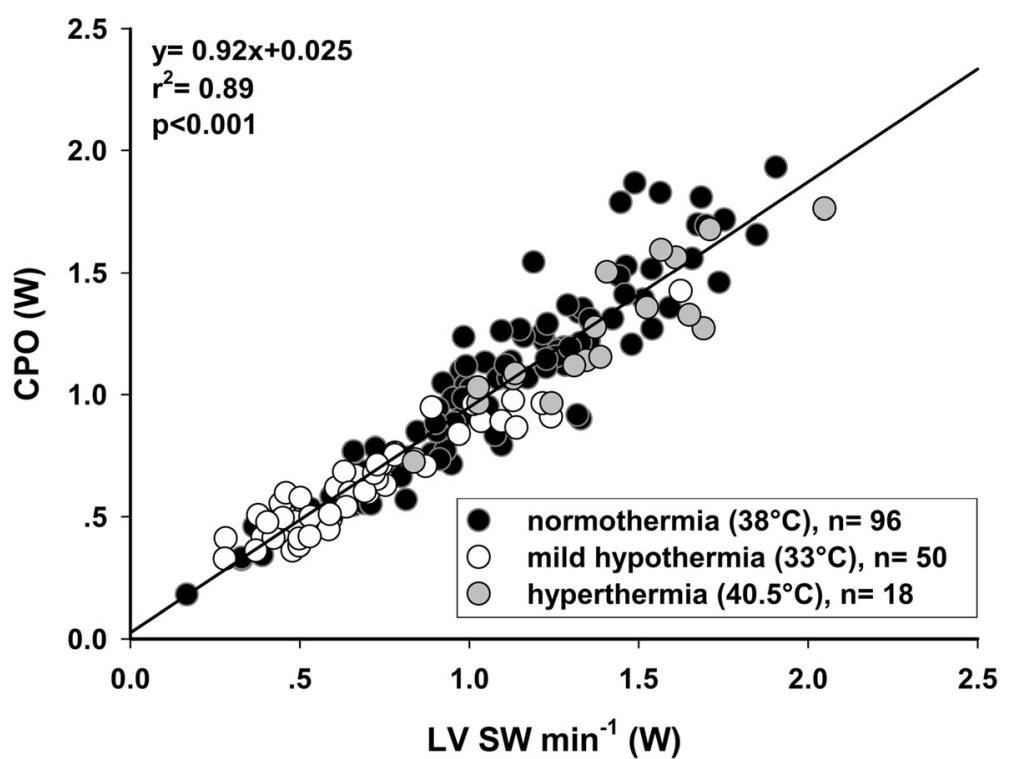

Fig. 2 Cardiac Power Output (CPO) accurately reflects Left ventricular stroke work per minute (LV SW min ${ }^{-1}$ ) over a wide range of inotropic states. Any rise or fall of LV SW $\mathrm{min}^{-1}$ corresponds to an equivalent change of CPO

with LV SW (Fig. 3a; $r^{2}=0.02 ; p=0.059$ ) nor with LV SW $\min ^{-1}$ (Fig. 3b; $r^{2}=0.01 ; p=0.259$ ). Other common parameters of cardiac function showed a statistically significant but moderate correlation. RPP (Fig. 4a; $r^{2}=0.67 ; p<0.001$ ) did correlate better than TP (Fig. 4b; $r^{2}=0.54 ; p<0.001$ ) with LV SW $\min ^{-1}$. $\mathrm{LVP}_{\max }$ showed a similar correlation with LV SW (Fig. 5a; $r^{2}=0.55 ; p<0.001$ ), but correlated worse with LV SW $\min ^{-1}$ (Fig. 5b; $r^{2}=0.47 ; p<0.001$ ). LV $\mathrm{dP} / \mathrm{dt}_{\max }$ showed the worst correlation with both LV SW (Fig. 5c; $r^{2}=0.23 ; p<0.001$ ) and LV SW $\min ^{-1}$ (Fig. 5d; $\left.r^{2}=0.28 ; p<0.001\right)$. An additional file shows the correlations between CPO and LV SW $\mathrm{min}^{-1}$ of each individual experimental group (Additional file 1).

\section{Discussion}

Here, we demonstrate that Cardiac Power Output accurately reflects left ventricular stroke work per minute over a wide range of inotropic states. Data were collected from experimental studies of the hemodynamic impact of mild hypothermia on acute heart failure models, such as 1) resuscitation after ventricular fibrillation, 2) coronary no-reflow infarction, 3) endotoxemia by LPS-infusion, 4) sevofluraneinduced myocardial depression and 5) beta-adrenergic stimulation via dobutamine infusion at different core-body temperatures. We therefore investigated conditions ranging from hypo- to hypercontractile LV function. CPO showed a high positive correlation over the whole range of inotropic states with $\mathrm{LV} \mathrm{SW} \mathrm{min}^{-1}$, making it a valid parameter to monitor inotropic interventions in the ICU setting.
Cardiac power output and left ventricular stroke work The correlation between CPO, as measured by pulmonary artery catheterization (PAC) and invasive arterial pressure, and LV SW $\mathrm{min}^{-1}$ measured via the invasive gold-standard (conductance method) has never been tested so far. Since pressure-volume analysis remains a time-consuming, complex and highly invasive measurement requiring additional equipment and expertise, the concept of deriving similar information by assessing CPO instead of LV SW is therefore quite attractive for the clinical practice. Being assessed as the product of cardiac output and mean aortic pressure and therefore combining the heart's ability to create both pressure and flow, CPO describes the function of the heart as a hydraulic pump [13]. More precisely, hydraulic power in general consists of two components. The power expended to generate a steady (or non-pulsatile) flow is described as the mean external power, which is assessed as the product of mean arterial pressure and cardiac output. The energy used in producing the pulsatile component of flow and pressure is described as pulsatile power. The sum of both mean and pulsatile power results in the total external hydraulic power generated by the ventricle [33]. Hence, CPO is a measure of mean power, whereas LV SW is a measure of total energy imparted to the vasculature system including pulsatile power being assessed on a beat to beat basis. Under physiological conditions, the fraction of pulsatile power is about $10 \%$ of the total power generated by the left 


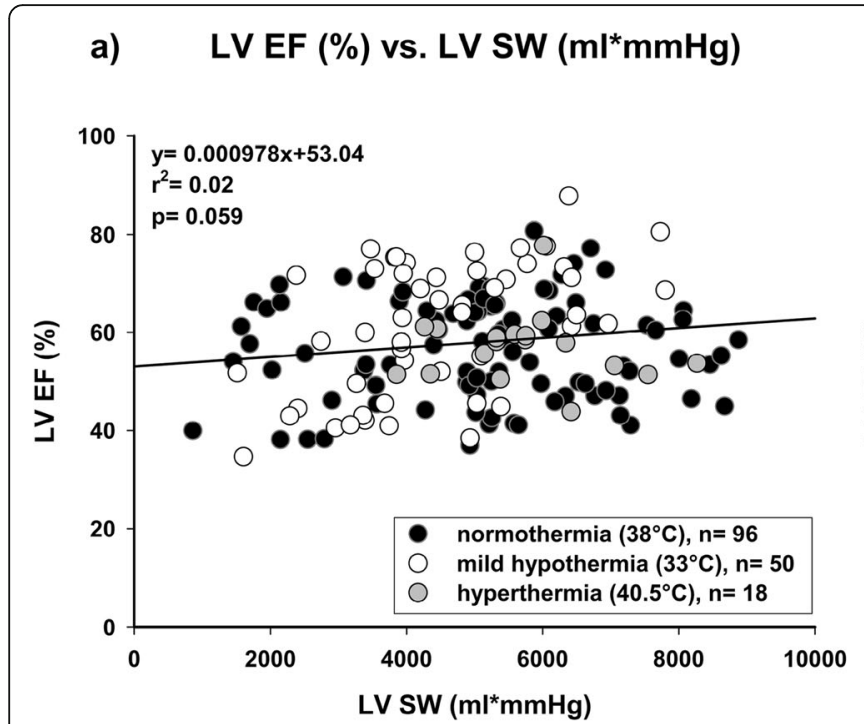

b) LV EF (\%) vs. LV SW $\mathrm{min}^{-1}$ (W)

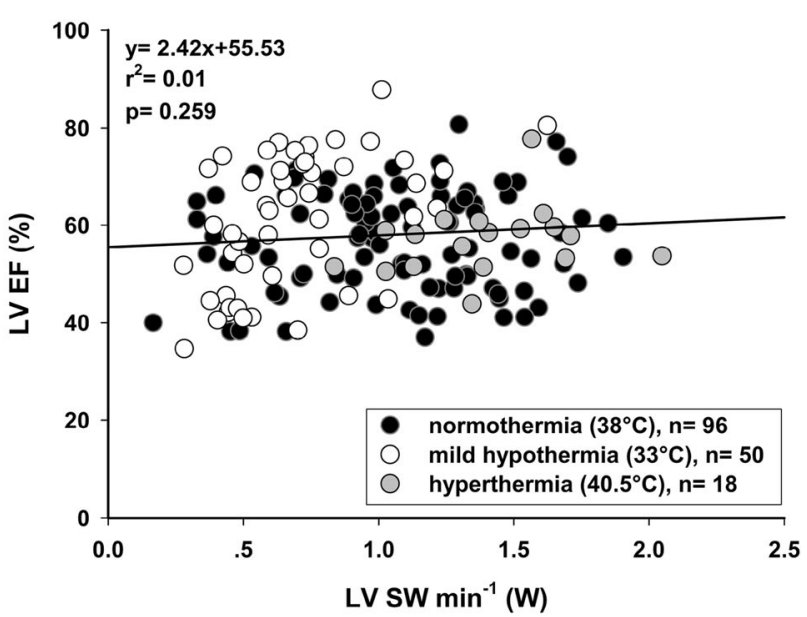

Fig. 3 Left ventricular ejection fraction (LV EF) did not correlate with (a) Left ventricular stroke work per beat (LV SW) nor with (b) Left ventricular stroke work per minute (LV SW $\mathrm{min}^{-1}$ )

ventricle, while it rises with arterial hypertension, decreased distensibility of the proximal arteries and increases in heart rate [34-36]. In the pulmonary circulation, pulsatile power can make up to $30 \%$ of total power [33, 37]. The ratio between pulsatile power and mean power can be regarded as a measure of the efficiency of ventricular-arterial coupling, while an increase of the pulsatile fraction indicates a decrease in efficiency [35]. Although CPO does not include information of pulsatile power, we could show here for the first time a strong correlation to LV SW $\mathrm{min}^{-1}$ under various inotropic states during acute heart failure. Of note, none of the experiments included LV output tract (LVOT) obstruction or aortic valve obstruction as well as severe stiffness of the aorta, which in turn would have increased LV SW min $^{-1}$ without changing CPO.

As abovementioned, CPO was shown to be the best predictor of intrahospital mortality in patients with cardiogenic shock [12]. Furthermore, it allows an exact hemodynamic characterization of patients with acute congestive heart failure by plotting CPO with SVR [38] and it was shown to be the only parameter, when measured at baseline, with a significant prediction of

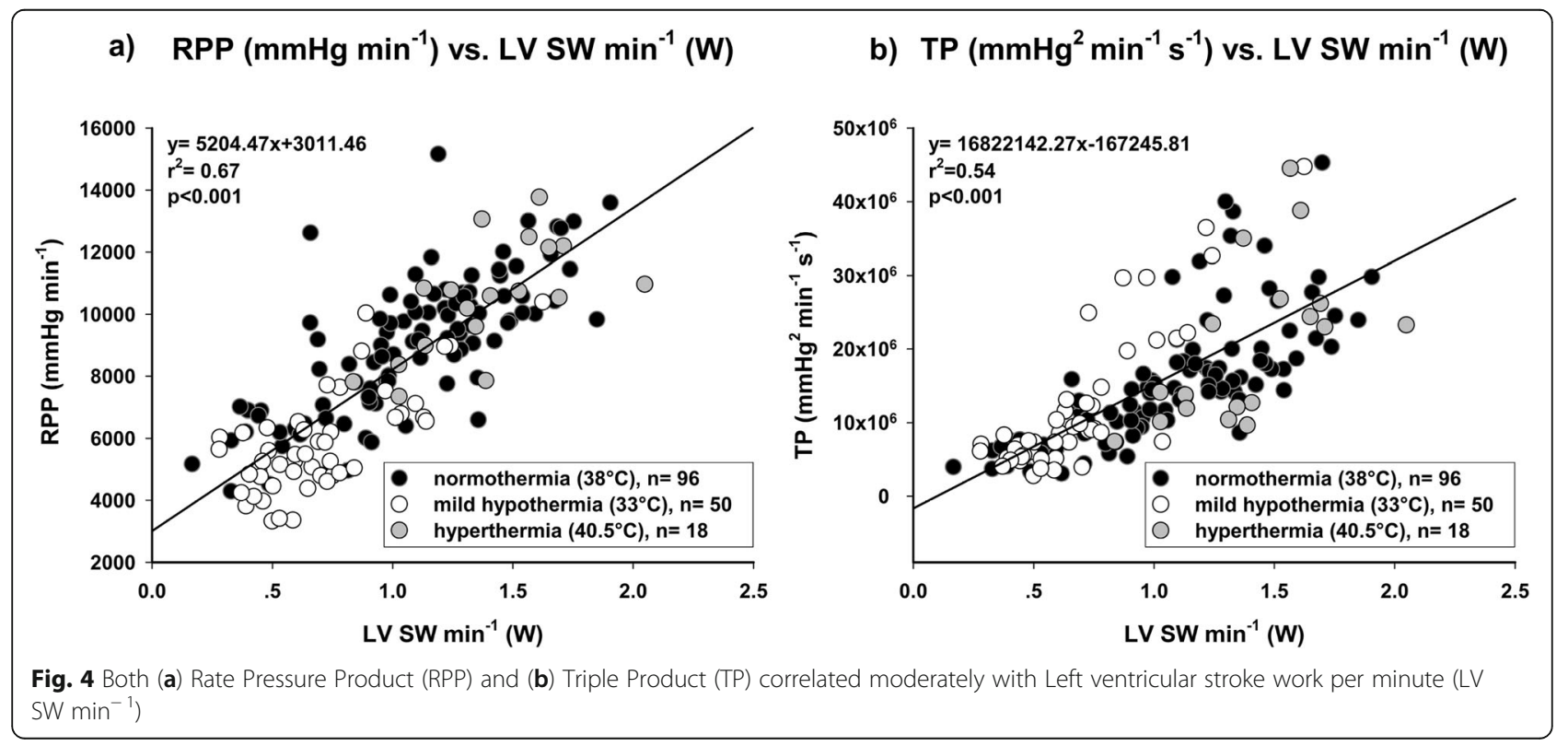


a) LVPmax $(\mathrm{mmHg})$ vs. LV SW $\left(\mathrm{ml}^{*} \mathrm{mmHg}\right)$

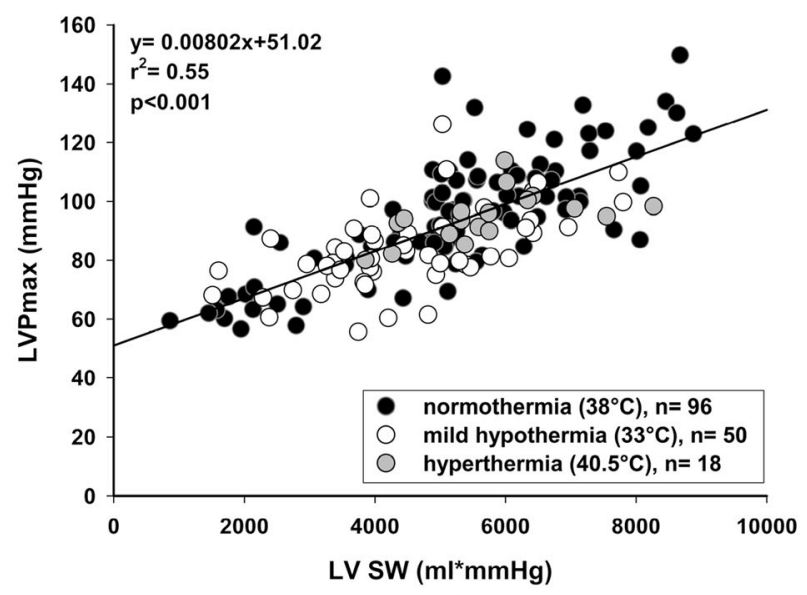

c) LV dP/dTmax $\left(\mathrm{mmHg} \mathrm{s}^{-1}\right)$ vs. LV SW $\left(\mathrm{ml}^{*} \mathrm{mmHg}\right)$

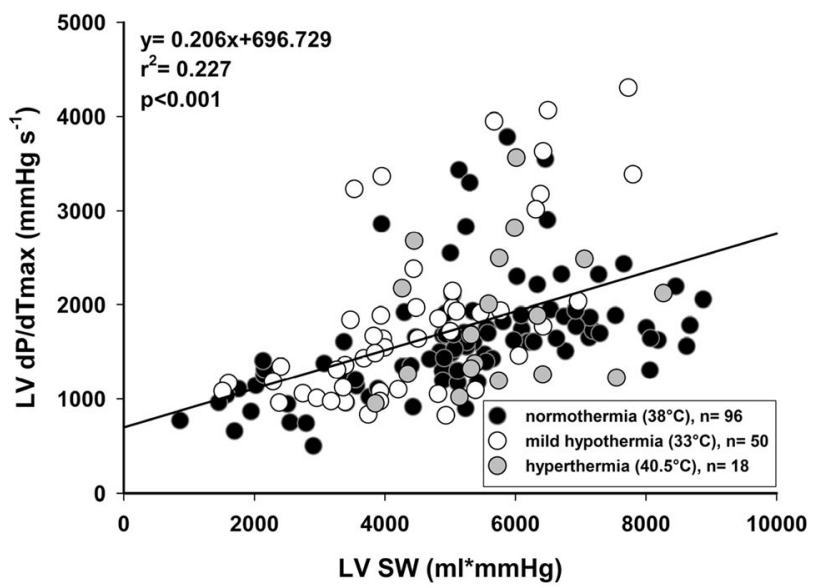

b) LVPmax (mmHg) vs. LV SW $\min ^{-1}(\mathrm{~W})$

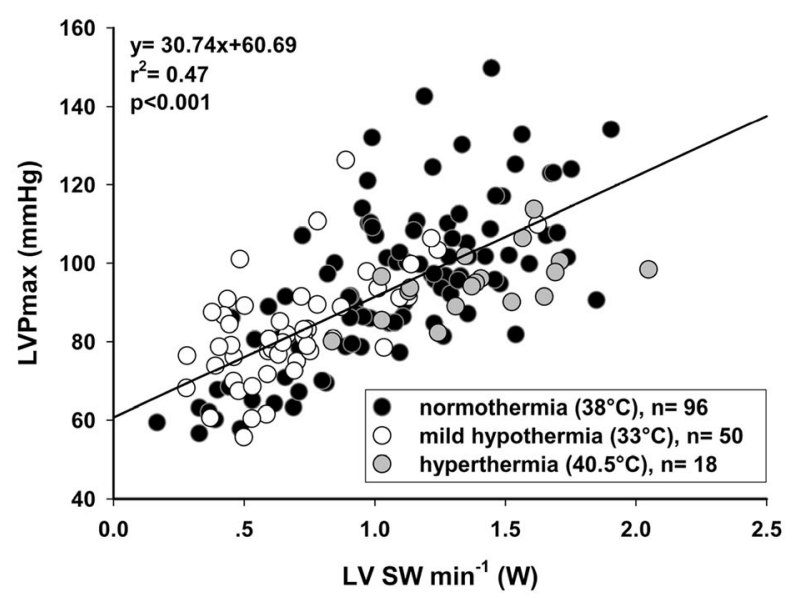

d) LV dP/dTmax $\left(\mathrm{mmHg} \mathrm{s}^{-1}\right)$ vs. LV SW $\min ^{-1}$ (W)

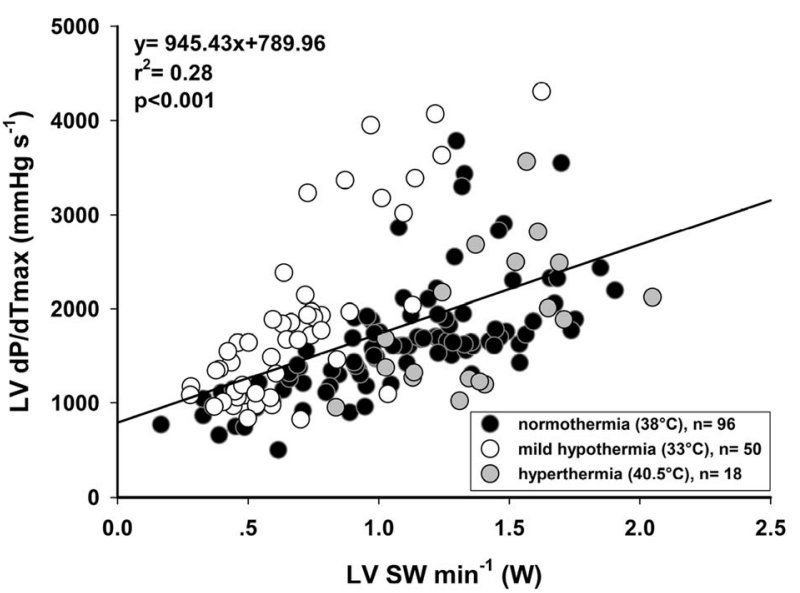

Fig. 5 LV maximum pressure (LVP $\max$ ) showed a better correlation with both (a) Left ventricular stroke work per beat and (b) Left ventricular stroke work per minute (LV SW min $\left.^{-1}\right)$ than maximum rate of rise of LVP (LV dP/dT $\max _{\text {, }} \mathbf{c}$ and $\mathbf{d}$ )

recurring severe acute heart failure (manifested as frank pulmonary edema) within $24 \mathrm{~h}$ from hospitalization in the ICU setting [39]. However, the role of CPO has not been yet fully integrated into the clinical ICU routine, in part due to a lack of data supporting the use of PAC in patients with advanced heart failure. In a meta-analysis investigating 13 randomized controlled trials (RCT) on the impact of PAC on survival, Shah et al. [4] argued that the neutral effects of PAC might be based on unclear hemodynamic goals in combination with a lack of effective treatment strategies driven by the obtained hemodynamic measurements $[2,4]$. We suggest here that $\mathrm{CPO}$ obtained via PAC is an excellent parameter to monitor cardiac performance in experimental conditions resembling critically-ill patients. Furthermore, recent data show the feasibility of a minimally invasive assessment of CPO and cardiac power integral [40-43], opening new scenarios of CPO-based monitoring of critical patients without the need for PAC.

\section{Left ventricular ejection fraction}

We could clearly show that LV EF does not reflect external cardiac work over a wide range of experimental inotropic states representative for patients during acute heart failure. In clinical practice, LV EF plays a major role in the diagnosis and treatment of heart failure, as a surrogate marker of cardiac remodelling [44] and reverse remodelling [45] or as a general predictor of outcome in heart failure with reduced LV EF [46, 47]. Nevertheless, our data imply that LV EF is not accurate enough in mirroring the function of the heart as a hydraulic pump under various conditions of acute heart failure and should be preferably interpreted within the hemodynamic context. 


\section{LV pressure and pressure-derived indices of cardiac workload}

The rate pressure product and the triple product are both clinical indices of myocardial oxygen consumption and therefore regarded as indirect measures of cardiac work [48-52]. Thus, we used these parameters as surrogates for LV SW $\mathrm{min}^{-1}$. In our findings, we confirm a correlation between both RPP and TP with LV SW min $^{-}$ 1, with RPP having a better correlation than TP. The worse correlation of TP with LV SW $\min ^{-1}$ could be explained by integrating $\mathrm{LV} \mathrm{dP} / \mathrm{dt}_{\max }$ into the calculation which is known to be a preload, afterload and heart rate dependent measure of cardiac contractility [44, 53-55]. Also, LV $\mathrm{dP} / \mathrm{dt}_{\max }$ itself correlated worst with $\mathrm{LV} \mathrm{SW}$ and LV SW $\min ^{-1}$.

Interestingly, $\mathrm{LVP}_{\max }$ alone correlated as good as the TP with LV SW, emphasizing the potential role of estimating LV pressure as a measure of the actual work done by the left ventricle in specific subpopulations of cardiogenic shock patients, i.e. under left ventricular assistance.

\section{Limitations}

The main limitation is the retrospective design of the study, meaning that the analysed data were not always part of the major outcome of the original studies. However, the quality of the original tracings has been reviewed by 2 independent investigators. Specific limitations related to the experimental setup from which these data were derived have been described earlier [21-24]. Another limitation of the study is related to the fact that animals were investigated under general anaesthesia, in order to minimize the animals' distress and to obtain stable hemodynamic conditions. Nevertheless, we believe the data to be representative enough for the translation to a clinical ICU setting. Furthermore, the study did not include experimental models of LVOT obstruction or increased stiffness of the aorta for modelling of patients with aortic stenosis or severe arteriosclerosis.

\section{Conclusions}

$\mathrm{CPO}$ is an excellent parameter of external cardiac work over a wide range of inotropic states, while clinically established indices to describe cardiac function, such as LV ejection fraction, rate-pressure product and triple product perform poorly.

These data further support the use of CPO to monitor cardiac workload as well as the effect of inotropic interventions in critically ill patients in the ICU setting. Clinical studies will address the impact of CPO on improving patients' survival.

\section{Supplementary information}

Supplementary information accompanies this paper at https://doi.org/10. 1186/s12872-019-1212-2.

Additional file 1. Individual correlations of CPO (W) vs. LVSW $\min ^{-1}$ (W) Cardiac Power Output (CPO) significantly reflects Left ventricular stroke work per minute (LV SW min-1) over a wide range of inotropic states in each experimental group (Group 1-5). Groups are described as follows: a) group 1: resuscitation after ventricular fibrillation (RES); b) group 2: myocardial infarction by coronary microembolisation (CME); c) group 3: endotoxemia by LPS-infusion (LPS); d) group 4: sevoflurane-induced

myocardial depression (SEVO); e) group 5: Temperature modulation from hyperthermia to mild hypothermia vs dobutamine (DOB). Any rise or fall of LV SW min-1 corresponds to an equivalent change of CPO.

\section{Abbreviations}

CME: Coronary microembolisation; CO: Cardiac output; CPO: Cardiac power output; DOB: Temperature modulation from hyperthermia to mild hypothermia vs dobutamine; $\mathrm{FiO}_{2}$ : Fraction of inspired oxygen; HT: Hyperthermia; I:E: Ratio of inspiration to expiration; ICU: Intensive-care unit; LPS: Lipopolysaccharide; LV dP/dt max: Maximal rate of rise of LVP; LV EF: LV ejection fraction; LV SW: Left ventricular stroke work; LVOT: LV output tract; LVP max: LV maximum pressure; MAP: Mean aortic pressure; MH: Mild hypothermia; NT: Normothermia; PAC: Pulmonary artery catheterization; RCT: Randomized controlled trial; RES: Resuscitation after ventricular fibrillation; ROSC: Return of spontaneous circulation; RPP: Rate-pressure product; $\mathrm{S}_{2} \mathrm{O}_{2}$ : Arterial oxygen saturation; SEVO: Sevoflurane-induced myocardial depression; TP: Triple product; VF: Ventricular fibrillation; VT: Tidal volume

\section{Acknowledgements}

Not applicable.

\section{Authors' contributions}

$D A, A F, M S, M M, D Z, K P K, J V, B Z, H M, P S, B P, H P, A A$ : conception and design of research; MM, DZ, JV, BZ, HP, AA: performed experiments; DA, AF, MS, HP, AA: analyzed data; DA, AF, MS, MM, DZ, KPK, JV, BZ, HM, PS, BP, HP, AA: Interpreted results of experiments; DA, AF, AA: prepared figures; DA, AA, HP: drafted manuscript; all authors edited and revised the manuscript and approved its final version.

\section{Funding}

$\mathrm{AA}$ is a participant in the $\mathrm{BIH}$-Charité Clinician Scientist Program funded by the Charité - Universitätsmedizin Berlin and the Berlin Institute of Health. AA and HP were supported by the Horizon 2020 CUPIDO project GA 720834 during study design, data analysis, interpretation of data and in writing the manuscript. The funding body had no impact on data collection.

\section{Availability of data and materials}

The datasets used and/or analysed during the current study are available from the corresponding author on reasonable request.

\section{Ethics approval and consent to participate}

The experiments were performed either at the Section for Surgical Research, Department of Experimental Surgery or at the Institute for Biomedical Research of the Medical University of Graz (Auenbruggerplatz 15, 8036 Graz, Austria). The experimental protocols were approved by the local bioethics committee of Vienna, Austria (Austrian Committee for Animal Trials, in German "Tierversuchskommission", BMWF-66.010/0091-II/3b/2013, BMWF66.010/0033-II/10b/2008, BMWF-66.010/0103-II/10b/2009, BMWF-66.010/0108II/3b/2010), and conforms to the "European Convention for the Protection of Vertebrate Animals used for Experimental and other Scientific Purposes" (Council of Europe No 123, Strasbourg 1985).

Consent for publication

Not applicable.

\section{Competing interests}

None of the authors reports a relationship with industry and other relevant entities - financial or otherwise - that might pose a conflict of interest in 
connection with the submitted article. The following authors report financial activities outside the submitted work: DA reports no conflict of interest. AF reports no conflict of interest. BP reports having received consultancy and lecture honoraria from Bayer, Daiichi Sankyo, MSD, Novartis, Sanofi-Aventis, Stealth Peptides and Vifor Pharma; and editor honoraria from the Journal of the American College of Cardiology. HP reports no conflict of interest. AA reports no conflict of interest. MM reports no conflict of interest. DZ reports no conflicts of interest. KPK reports having received lecture honoraria from Bristol-Myers Squibb.

\section{Author details}

'Department of Internal Medicine and Cardiology, Charité -

Universitätsmedizin Berlin, Campus Virchow-Klinikum, Augustenburgerplatz 1 , 13353 Berlin, Germany. ${ }^{2}$ Berlin Institute of Health (BIH), Berlin, Germany. ${ }^{3}$ DZHK (German Centre for Cardiovascular Research), partner site Berlin, Berlin, Germany. ${ }^{4}$ Department of General and Interventional Cardiology, University Heart Center Hamburg-Eppendorf Martinistr 52, 20246 Hamburg, Germany. ${ }^{5}$ DZHK (German Centre for Cardiovascular Research), partner site Hamburg/Kiel/Lübeck, Hamburg, Germany. ${ }^{6}$ Department of Internal Medicine, Division of Cardiology, Medical University of Graz , Auenbruggerplatz 15, 8036 Graz, Austria. ${ }^{7}$ Department of Cardiology, Heart Center Leipzig at University of Leipzig, Leipzig, Germany. ${ }^{8}$ Leipzig Heart Institute at Heart Center Leipzig, Leipzig, Germany. ${ }^{9}$ Department of Cardiothoracic Surgery, Medical University of Graz Auenbruggerplatz 29, 8036 Graz, Graz, Austria. ${ }^{10}$ Department of Cardiology, Leiden University Medical Center, PO 9600, 2300 RC Leiden, The Netherlands. ${ }^{11}$ Department of Internal Medicine and Cardiology, German Heart Center Berlin, Berlin, Germany.

${ }^{12}$ Department of Cardiology, Contilia Heart and Vessel Centre, St.

Marien-Hospital Mülheim, 45468 Mülheim, Germany.

Received: 5 June 2019 Accepted: 26 September 2019

Published online: 15 October 2019

\section{References}

1. Schwann NM, Hillel Z, Hoeft A, Barash P, Möhnle P, Miao Y, et al. Lack of effectiveness of the pulmonary artery catheter in cardiac surgery. Anesth Analg. 2011;113:994-1002.

2. Binanay C, Califf RM, Hasselblad V, O'Connor CM, Shah MR, Sopko G, et al. Evaluation study of congestive heart failure and pulmonary artery catheterization effectiveness: the ESCAPE trial. JAMA. 2005;294:1625-33.

3. Harvey S, Harrison DA, Singer M, Ashcroft J, Jones CM, Elbourne D, et al. Assessment of the clinical effectiveness of pulmonary artery catheters in management of patients in intensive care (PAC-man): a randomised controlled trial. Lancet. 2005:366:472-7.

4. Shah MR, Hasselblad V, Stevenson LW, Binanay C, O'Connor CM, Sopko G, et al. Impact of the pulmonary artery catheter in critically ill patients. JAMA. 2005;294:1664

5. Yu DT, Platt R, Lanken PN, Black E, Sands KE, Schwartz JS, et al. Relationship of pulmonary artery catheter use to mortality and resource utilization in patients with severe sepsis*. Crit Care Med. 2003;31:2734-41.

6. Connors AF, Speroff T, Dawson NV, Thomas C, Harrell FE, Wagner D, et al. The effectiveness of right heart catheterization in the initial care of critically ill patients. SUPPORT investigators. JAMA. 1996;276:889-97.

7. Cohen MG, Kelly RV, Kong DF, Menon V, Shah M, Ferreira J, et al. Pulmonary artery catheterization in acute coronary syndromes: insights from the GUSTO IIb and GUSTO III trials. Am J Med. 2005;118:482-8.

8. Chittock DR, Dhingra VK, Ronco JJ, Russell JA, Forrest DM, Tweeddale M, et al. Severity of illness and risk of death associated with pulmonary artery catheter use*. Crit Care Med. 2004;32:911-5.

9. Richard C, Warszawski J, Anguel N, Deye N, Combes A, Barnoud D, et al. Early use of the pulmonary artery catheter and outcomes in patients with shock and acute respiratory distress syndrome: a randomized controlled trial. JAMA. 2003;290:2713-20.

10. Yancy CW, Jessup M, Bozkurt B, Butler J, Casey DE, Drazner MH, et al. 2013 ACCF/AHA guideline for the Management of Heart Failure: executive summary. Circulation. 2013;128:1810-52

11. Ponikowski P, Voors AA, Anker SD, Bueno H, Cleland JGF, Coats AJS, et al. 2016 ESC guidelines for the diagnosis and treatment of acute and chronic heart failure. Eur Heart J. 2016;37:2129-200.
12. Fincke R, Hochman JS, Lowe AM, Menon V, Slater JN, Webb JG, et al. Cardiac power is the strongest hemodynamic correlate of mortality in cardiogenic shock: a report from the SHOCK trial registry. J Am Coll Cardiol. 2004:44:340-8.

13. Cotter G, Williams SG, Vered Z, Tan LB. Role of cardiac power in heart failure. Curr Opin Cardiol. 2003;18:215-22.

14. Williams SG, Cooke GA, Wright DJ, Parsons WJ, Riley RL, Marshall P, et al. Peak exercise cardiac power output; a direct indicator of cardiac function strongly predictive of prognosis in chronic heart failure. Eur Heart J. 2001;22: 1496-503.

15. Burkhoff D, Mirsky I, Suga H. Assessment of systolic and diastolic ventricular properties via pressure-volume analysis: a guide for clinical, translational, and basic researchers. Am J Physiol Heart Circ Physiol. 2005;289:H501-12.

16. Kass DA, Yamazaki T, Burkhoff D, Maughan WL, Sagawa K. Determination of left ventricular end-systolic pressure-volume relationships by the conductance (volume) catheter technique. Circulation. 1986;73:586-95.

17. Kass DA, Midei M, Graves W, Brinker JA, Maughan WL. Use of a conductance (volume) catheter and transient inferior vena caval occlusion for rapid determination of pressure-volume relationships in man. Catheter Cardiovasc Diagn. 1988;15:192-202.

18. Suga H. Ventricular energetics. Physiol Rev. 1990;70:247-77.

19. Suga $\mathrm{H}$. Total mechanical energy of a ventricle model and cardiac oxygen consumption. Am J Phys. 1979;236:H498-505.

20. Baan J, van der Velde ET, de Bruin HG, Smeenk GJ, Koops J, van Dijk AD, et al. Continuous measurement of left ventricular volume in animals and humans by conductance catheter. Circulation. 1984;70:812-23.

21. Schwarzl M, Steendijk P, Huber S, Truschnig-Wilders M, Obermayer-Pietsch $\mathrm{B}$, Maechler $\mathrm{H}$, et al. The induction of mild hypothermia improves systolic function of the resuscitated porcine heart at no further sympathetic activation. Acta Physiol (Oxford). 2011;203:409-18.

22. Schwarzl M, Huber S, Maechler H, Steendijk P, Seiler S, Truschnig-Wilders M, et al. Left ventricular diastolic dysfunction during acute myocardial infarction: effect of mild hypothermia. Resuscitation. 2012;83:1503-10.

23. Schwarzl M, Seiler S, Wallner M, von Lewinski D, Huber S, Maechler $H$, et al. Mild hypothermia attenuates circulatory and pulmonary dysfunction during experimental endotoxemia. Crit Care Med. 2013:41:e401-10.

24. Alogna A, Manninger M, Schwarzl M, Zirngast B, Steendijk P, Verderber J, et al. Inotropic effects of experimental hyperthermia and hypothermia on left ventricular function in pigs-comparison with Dobutamine. Crit Care Med. 2016;44:e158-67.

25. Schwarzl M, Alogna A, Zirngast B, Steendijk P, Verderber J, Zweiker D, et al. Mild hypothermia induces incomplete left ventricular relaxation despite spontaneous bradycardia in pigs. Acta Physiol (Oxford). 2015;213:653-63.

26. Steendijk P, Staal E, Jukema JW, Baan J. Hypertonic saline method accurately determines parallel conductance for dual-field conductance catheter. Am J Physiol Heart Circ Physiol. 2001;281:H755-63.

27. Post H, Schmitto JD, Steendijk P, Christoph J, Holland R, Wachter R, et al. Cardiac function during mild hypothermia in pigs: increased inotropy at the expense of diastolic dysfunction. Acta Physiol (Oxford). 2010;199:43-52.

28. Heier T, Caldwell JE. Impact of hypothermia on the response to neuromuscular blocking drugs. Anesthesiology. 2006;104:1070-80.

29. Heier T, Caldwell JE, Sessler DI, Miller RD. Mild intraoperative hypothermia increases duration of action and spontaneous recovery of vecuronium blockade during nitrous oxide-isoflurane anesthesia in humans. Anesthesiology. 1991;74:815-9.

30. Caldwell JE, Heier T, Wright PM, Lin S, McCarthy G, Szenohradszky J, et al. Temperature-dependent pharmacokinetics and pharmacodynamics of vecuronium. Anesthesiology. 2000;92:84-93.

31. Leslie K, Sessler DI, Bjorksten AR, Moayeri A. Mild hypothermia alters propofol pharmacokinetics and increases the duration of action of atracurium. Anesth Analg. 1995;80:1007-14.

32. Aranake A, Mashour GA, Avidan MS. Minimum alveolar concentration: ongoing relevance and clinical utility. Anaesthesia. 2013;68:512-22.

33. Milnor WR, Conti CR, Lewis KB, O'Rourke MF. Pulmonary arterial pulse wave velocity and impedance in man. Circ Res. 1969;25:637-49.

34. O'Rourke MF. Steady and pulsatile energy losses in the systemic circulation under normal conditions and in simulated arterial disease. Cardiovasc Res. 1967;1:313-26

35. Nichols WW, O'Rourke MF, Avolio AP, Yaginuma T, Pepine CJ, Conti CR. Ventricular/vascular interaction in patients with mild systemic hypertension and normal peripheral resistance. Circulation. 1986;74:455-62. 
36. Abel FL. Fourier analysis of left ventricular performance. Evaluation of impedance matching. Circ Res. 1971;28:119-35.

37. Laskey WK, Ferrari VA, Palevsky HI, Kussmaul WG. Ejection characteristics in primary pulmonary hypertension. Am J Cardiol. 1993;71:1111-4.

38. Cotter G, Moshkovitz Y, Kaluski E, Milo O, Nobikov Y, Schneeweiss A, et al. The role of cardiac power and systemic vascular resistance in the pathophysiology and diagnosis of patients with acute congestive heart failure. Eur J Heart Fail. 2003;5:443-51.

39. Cotter G, Moshkovitz Y, Milovanov O, Salah A, Blatt A, Krakover R, et al. Acute heart failure: a novel approach to its pathogenesis and treatment. Eur J Heart Fail. 2002;4:227-34.

40. Rimehaug AE, Lyng O, Nordhaug DO, Løvstakken L, Aadahl P, KirkebyGarstad I. Cardiac power integral: a new method for monitoring cardiovascular performance. Phys Rep. 2013;1:e00159.

41. Nakayama M, Chen CH, Nevo E, Fetics B, Wong E, Kass DA. Optimal preload adjustment of maximal ventricular power index varies with cardiac chamber size. Am Heart J. 1998;136:281-8.

42. Sharir T, Feldman MD, Haber H, Feldman AM, Marmor A, Becker LC, et al. Ventricular systolic assessment in patients with dilated cardiomyopathy by preload-adjusted maximal power: validation and noninvasive application. Circulation. 1994;89:2045-53.

43. Schmidt C, Roosens C, Struys M, Deryck YL, Van Nooten G, Colardyn F, et al. Contractility in humans after coronary artery surgery. Anesthesiology. 1999;91:58-70.

44. Kass D, Maughan WL, Guo ZM, Kono A, Sunagawa K, Sagawa K. Comparative influence of load versus inotropic states on indexes of ventricular contractility: experimental and theoretical analysis based on pressure-volume relationships. Circulation. 1987;76:1422-36.

45. MacMahon S, Sharpe N. Randomised, placebo-controlled trial of carvedilol in patients with congestive heart failure due to ischaemic heart disease. Australia/New Zealand Heart Failure Research Collaborative Group. Lancet (London, England). 1997;349:375-80.

46. Solomon SD, Anavekar N, Skali H, McMurray JJV, Swedberg K, Yusuf S, et al. Influence of ejection fraction on cardiovascular outcomes in a broad spectrum of heart failure patients. Circulation. 2005;112:3738-44.

47. Cohn JN, Johnson GR, Shabetai R, Loeb H, Tristani F, Rector T, et al. Ejection fraction, peak exercise oxygen consumption, cardiothoracic ratio, ventricular arrhythmias, and plasma norepinephrine as determinants of prognosis in heart failure. The V-HeFT VA Cooperative Studies Group. Circulation. 1993; $87(6$ Suppl):V15-16.

48. Gobel FL. Norstrom L a, Nelson RR, Jorgensen CR, Wang Y. the rate-pressure product as an index of myocardial oxygen consumption during exercise in patients with angina pectoris. Circulation. 1978;57:549-56.

49. Nelson RR, Gobel FL, Jorgensen CR, Wang K, Wang Y, Taylor HL. Hemodynamic predictors of myocardial oxygen consumption during static and dynamic exercise. Circulation. 1974;50:1179-89.

50. Katz $\mathrm{LN}$, Feinberg $\mathrm{H}$. The relation of cardiac effort to myocardial oxygen consumption and coronary flow. Circ Res. 1958;6:656-69.

51. Bernstein RD, Ochoa FY, Xu X, Forfia P, Shen W, Thompson Cl, et al. Function and Production of Nitric Oxide in the Coronary Circulation of the Conscious Dog During Exercise. Circ Res. 1996;79:840 LP-848.

52. Kitamura K, Jorgensen CR, Gobel FL, Taylor HL, Wang Y. Hemodynamic correlates of myocardial oxygen consumption during upright exercise. J Appl Physiol. 1972;32:516-22.

53. Mason DT, Braunwald E, Covell JW, Sonnenblick EH, Ross J. Assessment of cardiac contractility. The relation between the rate of pressure rise and ventricular pressure during isovolumic systole. Circulation. 1971;44:47-58.

54. Grossman W, Haynes F, Paraskos JA, Saltz S, Dalen JE, Dexter L. Alterations in preload and myocardial mechanics in the dog and in man. Circ Res. 1972;31:83-94

55. Miura T, Miyazaki S, Guth BD, Kambayashi M, Ross J. Influence of the forcefrequency relation on left ventricular function during exercise in conscious dogs. Circulation. 1992;86:563-71.

\section{Publisher's Note}

Springer Nature remains neutral with regard to jurisdictional claims in published maps and institutional affiliations.

\section{Ready to submit your research? Choose BMC and benefit from:}

- fast, convenient online submission

- thorough peer review by experienced researchers in your field

- rapid publication on acceptance

- support for research data, including large and complex data types

- gold Open Access which fosters wider collaboration and increased citations

- maximum visibility for your research: over $100 \mathrm{M}$ website views per year

At BMC, research is always in progress.

Learn more biomedcentral.com/submissions 